\title{
Sharing Our Successes
}

MOST OF OCEANOGRAPHY'S READERS are used to sharing their scientific successes in journal articles, by giving talks at scientific-society meetings, and through discussions with colleagues. But, wouldn't it be helpful to have a place to share some of your teaching successes, in particular, hands-on laboratories? Instructors of university-level oceanography courses might want to contribute to a collection of peerreviewed laboratories, or use some of them when working up next semester's curriculum. But, where do you share/find these labs? Well, you've come to the right place.

With the June issue of Oceanography, we launched a new column called "Hands-On Oceanography." Hands-on is broadly interpreted as those activities that actively engage students (i.e., activities where students have to make decisions, record results, and interpret results). The aim of this column is to provide peer-reviewed, high-quality laboratory templates for college- and graduate-level instructors to use and adapt for their classes. Keeping this in mind, the equipment and supplies needed for the lab should be relatively cheap to purchase and/or readily available. Activities that use real-time data and models in computer-based labs should also rely on technology generally available to university students. Once a lab is published in the magazine- and we expect to have a new one in every issue-we will share it via the TOS web site on the Hands-On page (go to http://www.tos.org/hands-on) and also in the issue's online archive.

We are in the early stages of implementing Hands-On Oceanography. We welcome comments and encourage submissions. In the future, on the Hands-On web page, we might add follow-up comments to a published lab, contributed by users who have suggestions on ways to enhance or simplify a lab, or who have more helpful instructions to add. We want to make the column and web page useful resources for you.

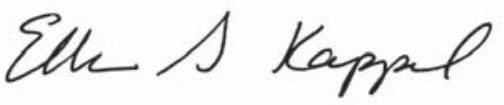

ELLEN S. KAPPEL, EDITOR

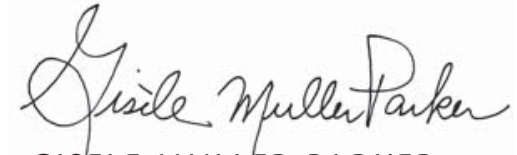

GISELE MULLER-PARKER, TOS EDUCATION COUNCILLOR 